\title{
Fundamentals of Three-Dimensional Digital Image Processing
}

\author{
J. Toriwaki and H. Yoshida
}

New York, NY: Springer, 2009, 272 pages, $\$ 89.95$

This book is a detailed description of the basics of 3-dimensional (3D) digital image processing, in particular the processing of images from techniques such as CT, MRI, and nuclear emission tomography. The book is based on 1980s research by the first author, Dr. Toriwaki, at Nagoya University and Chukyo University, Japan. The targeted audience is primarily graduate students and scientists in biomedical engineering and medical physics.

The book is divided into 7 chapters. The first part of the book talks about the geometric characteristics of 3D images, and the second part introduces algorithms for 3D image processing.

Chapter 1 provides an overview of 3D images and their processing. It covers the concept of continuous and digital 3D images and different types of 3D images. Chapter 2 begins with a formal and precise definition of 3D digital images. Image processing is defined algebraically as calculations on images, a mapping defined within an image space, or compositions of image calculations. From this background, the development of image-processing algorithms can be better understood. Chapter 3 introduces localized processing or filtering of 3D gray-scale images, with the focus on density value manipulations such as smoothing filters, difference filters, Laplacians, and region expansion. Most of these methods are direct expansions from their 2-dimensional counterparts and are relatively easy to understand.

Chapters 4 and 5 are the core of the book. Chapter 4 begins with a definition of connectivity and defines some fundamental concepts such as topology preservation conditions, Euler numbers, and path and distance functions, mainly on binary images. Some specific algorithms that compute these values are also included. Chapter 5 uses the concepts developed in chapter 4 to present algorithms for processing-connected components such as labeling, surface or axis thinning, and distance transformation. The authors detail the algorithms in a codelike presentation to allow easy translation from the algorithm to a workable program. The goal is to understand not only the context of the processing performed but also the structure and design methodology of the algorithm.

Chapter 6 covers the processing of connected components having density values as opposed to the processing of binary images as covered in chapter 4 . Chapter 6 discusses thinning, ridge line tracing, and gray-weighted distance transformations used on gray-scale images as examples of transformations required to incorporate density value information. The book ends with a description of 3D image visualization in chapter 7 , which uses techniques from computer graphics techniques such as ray casting for the generation of images. The techniques include ray tracing, volume rendering, and gradient shading for 3D image visualization. Ray tracing is important also for image reconstruction and radiation dose calculation.

An extensive reference list is provided at the end of the book.

I would like to congratulate the authors for making available this book detailing the steps of filtering, processing, and rendering medical images. If there were anything to be enhanced about the book in a future edition, it would be inclusion of a CD containing the example images and some programs in MATLAB (The MathWorks, Inc.) for the readers to get hands-on experience.

\section{Tinsu Pan}

M.D. Anderson Cancer Center

1515 Holcombe Blvd., 56

Houston, TX 77030

E-mail: tpan@mdanderson.org 